\title{
A importância da alimentação adequada para portadores de doenças inflamatórias intestinais e melhoria da qualidade de vida
}

The importance of proper nutrition for patients with inflammatory bowel diseases and improving quality of life

La importancia de una nutrición adecuada para los pacientes con enfermedades inflamatorias del intestino y la mejora de la calidad de vida

\author{
Priscila Cardoso Lima \\ ORCID: https://orcid.org/0000-0001-5692-6070 \\ Centro Universitário Fametro, Brasil \\ E-mail: priscil_a2@hotmail.com \\ Riane Loureiro Araújo de Carvalho \\ ORCID: https://orcid.org/0000-0001-5582-2667 \\ Centro Universitário Fametro, Brasil \\ E-mail: riane.lac@gmail.com \\ Thayná Macêdo de Oliveira \\ ORCID: https://orcid.org/0000-0003-1444-2590 \\ Centro Universitário Fametro, Brasil \\ E-mail: thaynamacedo2015@ @otmail.com \\ Francisca Marta Nascimento de Oliveira Freitas \\ ORCID: https://orcid.org/0000-0002-0044-0925 \\ Centro Universitário Fametro, Brasil \\ E-mail: Francisca.freitas@fametro.edu.br \\ Rebeca Sakamoto Figueiredo \\ ORCID: https://orcid.org/0000-0002-9819-8099 \\ Centro Universitário Fametro, Brasil \\ E-mail: rebeca.figueiredo@fametro.edu.br
}

\begin{abstract}
Resumo
Introdução: A Doença Inflamatória Intestinal (DII), corresponde a uma série de condições que envolvem a inflamação do trato gastrointestinal, principalmente no intestino, tornando-se vermelho, inchado e com presença de úlceras. Divide-se em dois grupos: Retocolite Ulcerosa e Doença de Crohn. Objetivo: Identificar a importância da estratégia alimentar adequada na promoção da qualidade de vida dos portadores de Doenças Inflamatórias Intestinais, quais são essas doenças e seus sintomas, qual a melhor estratégia nutricional para portadores de Doenças Inflamatórias Intestinais durante o tratamento e correlacionar a melhora da qualidade de vida com o uso da estratégia nutricional nos portadores de DII. Metodologia: Utilizou-se o método de pesquisa de caráter qualitativo. Resultados e discussão: As DII possuem tratamentos nos recursos de medicação, alimentação, suplementação nutricional, terapia convencional e em último caso, cirurgia para tentar solucionar a melhora dos sintomas em pacientes que não respondem às alternativas dos tratamentos oferecidos. Conclusão: A correlação direta da nutrição com a melhoria da qualidade de vida dos pacientes acometidos pelas doenças inflamatórias intestinais ainda possui escassez, porém, através das análises do tratamento com a equipe multidisciplinar é possível observar uma contribuição no quadro de estabilidade dos pacientes. $\mathrm{O}$ atendimento interdisciplinar, o olhar humanizado e integral dos profissionais da saúde para com os pacientes se faz necessário, assim como o desenvolvimento de novos estudos voltados para a temática.

Palavras-chave: Doenças inflamatórias intestinais; Doença de Crohn; Colite ulcerativa; Terapia nutricional; Qualidade de vida.

Abstract

Introduction: The Inflammatory Bowel Disease (IBD) corresponds to a series of conditions that involve inflammation of the gastrointestinal tract, mainly in the intestine, becoming red, swollen and with the presence of ulcers. It is divided into two groups: Ulcerative Colitis and Crohn's Disease. Objective: To identify the importance of an adequate food strategy in promoting the quality of life of patients with Inflammatory Bowel Diseases, what are these diseases and their symptoms, what is the best nutritional strategy for patients with Inflammatory Bowel Diseases during treatment and to correlate the improvement in quality of life with the use of the nutritional strategy in patients with IBD. Methodology: The qualitative research method was used. Results and discussion: The IBD have treatments in the resources of medication, food, nutritional supplementation, conventional therapy and, in the last case, surgery to
\end{abstract}


try to solve the improvement of symptoms in patients who do not respond to alternative treatments offered. Conclusion: The direct correlation of nutrition with the improvement in the quality of life of patients affected by inflammatory bowel diseases is still scarce, however, through the analysis of treatment with the multidisciplinary team, it is possible to observe a contribution to the stability of patients. Interdisciplinary care, the humanized and integral look of health professionals towards patients is necessary, as well as the development of new studies focused on the theme.

Keywords: Inflammatory bowel diseases; Crohn's disease; Ulcerative colitis; Nutritional therapy; Quality of life.

\section{Resumen}

Introducción: La Enfermedad Inflamatoria Intestinal (EII) corresponde a una serie de afecciones que involucran inflamación del tracto gastrointestinal, principalmente en el intestino, enrojeciéndose, inflamado y con presencia de úlceras. Se divide en dos grupos: colitis ulcerosa y enfermedad de Crohn. Objetivo: Identificar la importancia de una estrategia de alimentación adecuada en la promoción de la calidad de vida de los pacientes con Enfermedades Inflamatorias Intestinales, cuáles son estas enfermedades y sus síntomas, cuál es la mejor estrategia nutricional para los pacientes con Enfermedades Inflamatorias Intestinales durante el tratamiento y correlacionar la mejora de la calidad de vida con el uso de la estrategia nutricional en pacientes con EII. Metodología: Se utilizó el método de investigación cualitativa. Resultados y discusión: Las EII cuentan con tratamientos en los recursos de medicación, alimentación, complementación nutricional, terapia convencional y, en el último caso, cirugía para intentar solucionar la mejoría de los síntomas en los pacientes que no responden a los tratamientos alternativos ofrecidos. Conclusión: La correlación directa de la nutrición con la mejora en la calidad de vida de los pacientes afectados por enfermedades inflamatorias intestinales es aún escasa, sin embargo, a través del análisis del tratamiento con el equipo multidisciplinario, es posible observar un aporte a la estabilidad de los pacientes. . Es necesaria la atención interdisciplinaria, la mirada humanizada e integral de los profesionales de la salud hacia los pacientes, así como el desarrollo de nuevos estudios enfocados en la temática.

Palabras clave: Enfermedades inflamatorias del intestino; Enfermedad de Crohn; Colitis ulcerosa; Terapia nutricional; Calidad de vida.

\section{Introdução}

O presente artigo possui como escopo salientar a importância da alimentação adequada para portadores de doenças inflamatórias intestinais e melhoria da qualidade de vida, citando a epidemiologia, fisiopatologia, sendo ainda salientado a estratégia nutricional e tratamento, a assistência e o cuidado e como contribuem para a qualidade de vida, as questões socioemocionais implicadas e a melhora da qualidade de vida.

A Doença Inflamatória Intestinal (DII), corresponde a uma série de condições que envolvem a inflamação do trato gastrointestinal, principalmente no intestino, tornando-se vermelho, inchado e com presença de úlceras. Divide-se em dois grupos: Retocolite Ulcerosa (RU) e Doença de Crohn (DC) (Zanardi \& Nowacki, 2018). Apresentam-se de maneira idiopática e suas manifestações clínicas podem se estender por logos períodos. Apesar de terem essa correlação, são doenças com manifestações fisiopatológicas e clínicas distintas e que diferem tanto na evolução quanto na sensibilidade terapêutica sendo fundamental sua distinção durante o acompanhamento médico (Diretrizes Práticas da Organização Mundial de Gastroenterologia, 2009).

A Doença de Crohn é uma enfermidade inflamatória séria do trato gastrointestinal que afeta predominantemente a parte inferior do intestino delgado (Íleo) e intestino grosso (Cólon), mas pode afetar qualquer parte do trato gastrointestinal. A Doença de Crohn habitualmente causa diarreia, cólica abdominal, frequentemente febre e, às vezes, sangramento retal. Também podem ocorrer perda de apetite e perda de peso subsequente. Os sintomas variam de leve a grave, mas, em geral, as pessoas com Doença de Crohn podem ter vidas ativas e produtivas. (Hardt, 2012).

A retocolite ulcerativa (RCU) é uma doença inflamatória do Cólon (intestino grosso), de causa desconhecida e autoimune. Afeta predominantemente o cólon e o reto, resultando em diarreia crônica. (Cruz et al, 2012). Os sintomas podem incluir dor abdominal, urgência evacuatória, diarreia e sangue nas fezes. A inflamação começa no reto e pode se estender até o cólon de maneira contínua. Embora não haja uma cura conhecida, há muitas terapias efetivas para manter a inflamação sob controle (Añez, Fuenmayor \& Romero, 2012). 
O desenvolvimento e a origem das Doenças Inflamatórias Intestinais (DII) é multifatorial, podendo estar relacionada com a dieta de tipo ocidental, a qual é baseada no consumo de alimentos que são ricos em gorduras e açúcares. As doenças ocorrem com períodos de atividade e remissão. Muitos fatores podem ter um efeito benéfico na redução da frequência de atividades e no prolongamento do período de remissão, entre esses fatores há a alimentação (Farrukh \& Mayberry, 2014).

A terapia nutricional para uso das doenças inflamatórias intestinais (DII) tem como principal objetivo o controle dos sintomas, a prevenção, a correção da desnutrição e das diversas deficiências nutricionais, assim como a redução das sequelas em longo prazo, incluindo o déficit de crescimento em crianças e a osteoporose em adultos (Diestel et al, 2012).

Alguns fatores como estilo de vida, uso ou não de álcool e cigarro, exposição aos possíveis elementos patógenos intestinais, medicamentos, podem estar relacionados ao desenvolvimento dessas doenças (Pinho, 2008).

O artigo deve-se corroborar para os profissionais da saúde o quão necessário se faz o conhecimento sobre a importância da alimentação adequada para portadores de doenças inflamatórias intestinais e a melhoria da qualidade de vida

O presente artigo tem como objetivo geral identificar a importância da estratégia alimentar adequada na promoção da qualidade de vida dos portadores de Doenças Inflamatórias Intestinais e como objetivos específicos explicitar quais são as Doenças Inflamatórias Intestinais e seus sintomas, identificar qual a melhor estratégia nutricional para portadores de Doenças Inflamatórias Intestinais durante o tratamento e correlacionar a melhora da qualidade de vida com o uso da estratégia nutricional nos portadores de DII.

\section{Metodologia}

Para a análise de dados foi utilizada a pesquisa qualitativa a fim de argumentar os resultados do estudo por meio de análises e percepções de dados não-mensuráveis. De acordo com Deslauriers (1991), na pesquisa qualitativa, o aluno é sujeito e objeto da pesquisa simultaneamente, pois o conhecimento deve ser imparcial e limitado, já que o desenvolvimento da pesquisa é algo imprevisível.

Para a coleta de dados foram realizadas buscas em Livros, Revistas, Diretrizes Sociedades Brasileiras, artigos em sites como SciELo (Scientific Eletronic Library), PubMed (Servico da National Library of Medicine) e Google Acadêmico, utilizou-se os descritores: Doenças Inflamatórias Intestinais; Doença de Crohn; Colite Ulcerativa; Terapia Nutricional; Qualidade de vida, e suas combinações nas línguas portuguesa, espanhola e inglesa.

Para critérios de inclusão foram utilizadas referências entre 1991 a 2021, artigos com periódicos, sites e artigos acadêmicos que se enquadraram ao tema proposto. Foram analisados artigos acadêmicos, publicações de revistas acadêmicas, diretrizes, livros, para ser feito o tipo de estudo, de pesquisa, de revisão integrativa para o projeto.

Foram avaliados 70 citações e resumos relacionados a doenças gastrointestinais, Doenças Inflamatórias Intestinais; Doença de Crohn; Colite Ulcerativa; terapia nutricional e qualidade de vida obtidos a partir das revistas eletrônicas citadas. Porém, somente 43 foram aceitos. 
Quadro 1. Corpús do Estudo com os principais estudos selecionados.

\begin{tabular}{|c|c|c|c|c|}
\hline $\mathbf{N}^{\mathbf{o}}$ & ANO & TITULO DO ESTUDO & PERIÓDICO & AUTORES \\
\hline A1 & 2002 & $\begin{array}{l}\text { Prevalência de doenças crônicas auto-referidas e } \\
\text { utilização de serviços de saúde. }\end{array}$ & $\begin{array}{l}\text { Ciência e Saúde } \\
\text { Coletiva }\end{array}$ & $\begin{array}{l}\text { Almeida, M. F., } \\
\text { Barata, R. } \\
\text { Montero, } \\
\text { S. V., } \\
\text { Silva, Z. P. }\end{array}$ \\
\hline $\mathrm{A} 2$ & 2003 & $\begin{array}{l}\text { Opções terapêuticas para as doenças inflamatórias } \\
\text { intestinais: revisão. }\end{array}$ & $\begin{array}{ll}\text { Rev } & \text { Bras } \\
\text { Coloproct. } & \end{array}$ & $\begin{array}{l}\text { Biondo-Simões, M. } \\
\text { L. P., Mandelli, K. } \\
\text { K., Pereira, M. A. } \\
\text { C., \& Faturi, J. L. }\end{array}$ \\
\hline A3 & 2005 & $\begin{array}{l}\text { Perfil da doença de Crohn fistulizante em atividade em } \\
\text { dois serviços universitários em Salvador - Bahia. }\end{array}$ & $\begin{array}{l}\text { Revista Brasileira } \\
\text { de } \\
\text { Coloproctologia }\end{array}$ & $\begin{array}{lrr}\text { Andrade, } & \text { A. C. } & \text { M., } \\
\text { Santana, G. } & \text { O., } \\
\text { Santos, R. } & \text { R., } \\
\text { Guedes, J. C., } & \text { Lyra, L. G. C. }\end{array}$ \\
\hline A4 & 2007 & Sistema gastrointestinal. & Artmed & $\begin{array}{l}\text { Bya, L. M., \& } \\
\text { Krueger, G. R. F. }\end{array}$ \\
\hline A5 & 2010 & $\begin{array}{l}\text { Tratamento médico nutricional para doenças do trato } \\
\text { gastrointestinal inferior. }\end{array}$ & Elsevier & Beyer, P. L. \\
\hline A6 & 2010 & $\begin{array}{l}\text { Enfermagem médico-cirúrgica: perspectivas de saúde e } \\
\text { doença. }\end{array}$ & Lusodidacta & $\begin{array}{lr}\text { Frances, } & \text { D., } \\
\text { Monahan, } & \text { F., } \\
\text { Sharon, A. } & \end{array}$ \\
\hline A7 & 2012 & $\begin{array}{l}\text { Enfermedad inflamatoria intestinal: } \text { rectocolitis } \\
\text { úlcerosa idiopática y enfermedad de Crohn. }\end{array}$ & Revista GEN & $\begin{array}{l}\text { Añez, } \\
\text { Fuenmayor, M., \& } \\
\text { Romero, G. }\end{array}$ \\
\hline A8 & 2012 & $\begin{array}{l}\text { Epidemiological profile of } 175 \text { patients with Crohn's } \\
\text { disease submitted to biological therapy. }\end{array}$ & J Coloproctol. & Hardt, M. R., et al. \\
\hline A10 & 2012 & Crohn's disease. & Lancet & $\begin{array}{l}\text { Baumgart, D. C., \& } \\
\text { Sandborn, W. J. }\end{array}$ \\
\hline A11 & 2015 & $\begin{array}{l}\text { Doença inflamatória intestinal: Diagnóstico diferencial } \\
\text { na doença inflamatória intestinal. }\end{array}$ & Manole & $\begin{array}{l}\text { Cardozo, W. S., \& } \\
\text { Sobrado, C. W. }\end{array}$ \\
\hline A12 & 2016 & $\begin{array}{l}\text { Alimentação de qualidade para portadores de Doenças } \\
\text { Inflamatórias Intestinais (DII). }\end{array}$ & $\begin{array}{l}\text { revista Conexão } \\
\text { Eletrônica }\end{array}$ & Castro, L. S. D. \\
\hline A13 & 2018 & $\begin{array}{l}\text { Mudanças no consumo alimentar e nas condições } \\
\text { psicossociais geradas pela doença inflamatória } \\
\text { intestinal. }\end{array}$ & $\begin{array}{l}\text { Revista Brasileira } \\
\text { de Tecnologias } \\
\text { Sociais. }\end{array}$ & $\begin{array}{l}\text { Batista, TM; } \\
\text { Barretta, C., Matos, } \\
\text { C. H., et al. }\end{array}$ \\
\hline A14 & 2020 & $\begin{array}{l}\text { Métodos diagnósticos e terapêuticos das doenças } \\
\text { inflamatórias intestinais: revisão sistemática. }\end{array}$ & Para Res Med J. & $\begin{array}{l}\text { Barros, G. V. N., } \\
\text { Silva, T. S. S., Brito, } \\
\text { A. P. S. O., Garcia, } \\
\text { H. C. R., \& } \\
\text { Maneschy, R. B. }\end{array}$ \\
\hline A15 & 2021 & $\begin{array}{l}\text { Terapia nutricionais nas doenças inflamatórias } \\
\text { intestinais: Doença de Crohn e Retocolite Ulcerativa. }\end{array}$ & $\begin{array}{l}\text { Research, Society } \\
\text { and Development }\end{array}$ & $\begin{array}{l}\text { Santos, A. L. C., } \\
\text { Dias, B. C. O., } \\
\text { Silva, K. A., \& } \\
\text { Ferreira, J. C. S. }\end{array}$ \\
\hline
\end{tabular}

Fonte: Autores.

\section{Resultados e Discussão}

\subsection{Doenças inflamatórias intestinais}

\subsubsection{Epidemiologia}

A literatura, no que se refere a epidemiologia das DII, retrata que sua prevalência ocorre em pessoas brancas, na faixa etária de 20 a 40 anos, com o segundo pico da doença a partir dos 55 anos e com distribuições semelhantes em ambos os sexos, exceto na Doença de Crohn, em que o grupo de mulheres são mais frequentemente afetados. (Souza, Belasco \& AguilarNascimento, 2008).

Nos países em desenvolvimento onde as Doenças Inflamatórias Intestinais (DII) estão surgindo, a Retocolite Ulcerativa (RCU) tem uma maior frequência de incidência quando comparada à Doença de Crohn (DC). O Brasil ainda é considerado uma área de menor prevalência de DII, ainda que os registros literários dessas doenças tenham crescido 
significativamente no país. Nos últimos 20 anos, a DC geralmente excedeu a URC nas taxas de recorrência. (AMBCFM, 2008).

Discorrendo sobre a doença de Crohn e sobre a retocolite ulcerativa como entidades clínicas distintas, porém reconhecendo ambas como relacionadas e, que se incluem como pertencentes ao grupo das doenças intestinais inflamatórias. Um estudo descreveu sobre as doenças inflamatórias intestinais, a partir dos casos de internações registradas, em decorrência do agravamento da doença, em unidades de saúde, na macrorregião de saúde leste do Estado de Minas Gerais. Ele mostrou que as doenças inflamatórias intestinais têm sido consideradas um grande problema social, por gerarem repercussões impactantes na qualidade de vida dos portadores. Informando que, entre 1998 e 2005, foram registradas 363 internações em hospitais, por conta destas doenças inflamatórias, sendo 184 casos em homens e 179 casos em mulheres (Oliveira, Emerick \& Soares, 2010).

Seus resultados revelaram que na macrorregião leste do estado em questão, as internações por doenças inflamatórias intestinais têm representado $0,6 \%$ de todas as internações por conta de doenças do aparelho digestivo. Também que no período do estudo foram registradas 363 internações em hospitais por doenças inflamatórias intestinais, não sendo observada uma predominância de gênero, o que corrobora outros estudos internacionais (British Society Of Gastroenterology, 2003; Ekbom et al., 1991).

Com relação a faixa etária, $70 \%$ dos casos, acometeram pessoas entre 20 e 69 anos de idade. O estudo relatou dificuldades nos registros dos prontuários e o fato de que, com a exacerbação da doença, há uma tendência a procura de hospitais da rede privada (Almeida et al., 2002), o que dificultou o acompanhamento, em alguns casos. Durante o período, quatro óbitos foram registrados, cujo coeficiente de letalidade encontrou-se em cinco mortes a cada 1.000 (no caso das mulheres internadas) e, dez mortes a cada 1.000 (no caso de homens internados) (Oliveira, Emerick \& Soares, 2010).

Esses dados confirmam a baixa letalidade encontrada em outros estudos de Andrade et al (2005) em termos de tempo de internamento, observou-se um tempo médio de 6,5 dias, o que eleva os gastos do sistema de saúde.

No entanto, sobre a temática pode-se resumir que, há comprometimento dos dados epidemiológicos do sistema de informação de saúde por subnotificação e falta de padronização nos critérios diagnósticos, a exacerbação da doença eleva o tempo de internação com consequente impacto financeiro no sistema de saúde e que ainda é incipiente o número de estudos sobre a temática, que envolva mais variáveis, tais como: perfil dos pacientes portadores de doenças inflamatórias intestinais, alterações biopsicossociais que produz no paciente e na família, dentre outras (Oliveira, Emerick \& Soares, 2010).

\subsubsection{Fisiopatologia}

A Retocolite Ulcerativa (RCU) e a Doença de Crohn (DC) são doenças com algumas características fisiopatológicas e clínicas peculiares que as diferem tanto na evolução quanto na sensibilidade terapêutica, sendo, portanto, fundamental sua distinção durante acompanhamento médico (Maranhão, Vieira \& Campos, 2015).

A Retocolite Ulcerativa (RCU) se caracteriza pela inflamação crônica do cólon e reto, ficando confinada a essas regiões anatômicas. A inflamação se limita à camada mucosa e aparece de forma contínua e simétrica ao longo da porção afetada (Yashiro, 2014). Na Doença de Crohn (DC) as lesões não são contínuas, comprometendo em boa parte as camadas da parede e afetar qualquer parte do trato gastrointestinal (Maranhão, Vieira \& Campos, 2015).

Os sintomas mais apresentados em pacientes com RCU são dores abdominais e diarreia sanguinolenta, além da excreção retal de muco e pus, ocasionando na maioria das vezes mais de dez evacuações em períodos de crises da doença (fase ativa), prejudicando a qualidade de vida (Cavalcante et al., 2020).

A doença pode causar complicações ocorrendo megacólon tóxico, de forma que o intestino grosso fica com dificuldades de movimento devido a inflamação, ocasionando a distensão intestinal e a síndrome de resposta inflamatória 
sistêmica. Na inflamação crônica pode causar a estenose fibrótica intestinal ou desenvolver câncer associada à colite (Cavalcante et al., 2020).

A Doença de Crohn se caracteriza pela inflamação transmural, da mucosa à serosa, crônica do tubo digestório, podendo acometer qualquer porção deste, desde a boca até o ânus, embora haja predileção pelas regiões ileal ou ileocecal. O padrão da inflamação é salteado, ou seja, áreas de inflamação alternadas com áreas saudáveis, e gera reação granulomatosa não caseificante, além de ter a possibilidade de lesões aftoides na área acometida (Beyer, 2012).

Como as possibilidades de acometimento anatômico da DC são mais amplas, as manifestações clínicas podem variar conforme o local afetado. Quando confinadas ao intestino delgado, a diarreia e a dor abdominal são as manifestações mais comuns, sintomas constitucionais como perda ponderal e anemia também podem ocorrer (Frances, Monahan \& Sharon, 2010).

A diarreia pode ocorrer por má absorção, de nutrientes, fluidos ou eletrólitos, ou por exsudação de fluido intersticial para lúmen devido ao processo inflamatório. No acometimento do íleo mais distal, a má absorção de sais biliares pode ser o mecanismo da diarreia, permitindo que grandes quantidades destes sais cheguem ao intestino grosso e gerem maior secreção de cloro e de água pelo epitélio. Por fim, é importante ressaltar que é no íleo que ocorre absorção de vitamina B12, e sua escassez gera anemia megaloblástica, ou seja, outro mecanismo que gera anemia no contexto da DII (Bya \& Krueger, 2007).

As formas mais comuns das manifestações clínicas são a inflamatória, obstrutiva e/ou fistulizante. Diarreia crônica, dor abdominal, perda ponderal e sangramento retal fazem parte do quadro sintomático os principais sinais clínicos são a desnutrição, palidez, cutâneo-mucosa, dor, massa abdominal, distensão ou fistulização na parede abdominal (SBC, 2011).

\subsection{Estratégia nutricional e tratamento}

As DII possuem atualmente, tratamentos nos recursos de medicação, alimentação, suplementação nutricional, terapia convencional e em último caso, cirurgia para tentar solucionar a melhora dos sintomas em pacientes que não respondem às alternativas dos tratamentos oferecidos. Inicialmente, é necessário a investigação dos sintomas, o nível de gravidade e o tempo que o paciente vem apresentando tudo isso (Biondo-Simões et al, 2003).

O diagnóstico pode ser realizado através de anamnese detalhada da história pregressa, exame físico e sintomas. Atentar-se para os exames hemograma, velocidade de sedimentação, PCR, albumina, ferro sérico, ferritina, coproscopia e coprocultura, pois neles mostram-se a maioria dos níveis de inflamação do organismo e consequentemente, de alguma parte do intestino, excluindo dessa forma outros diagnósticos como causas virais, bacterianas, parasitárias ou outras comorbidades que possam apresentar anemia, por exemplo. Para completar o caso, é necessário o exame de íleo-colonoscópico e biópsia para definir a severidade e extensão da doença. Cujo é feito através do reto para avaliar o trato gastrointestinal, através de sedação e possui todo um preparo alimentar para que ele seja realizado. Tem importância para investigar se existe a presença de pólipos, qual o nível de inflamação, se superficial do reto e se há necessidade de fazer uma cirurgia de emergência por alguma parte comprometida do Trato Gastrointestinal. A partir dos resultados, é determinado um tratamento medicamentoso e alimentar para cada paciente. Apesar de ainda não existir um exame específico para diagnosticar qual exatamente é a DII, a colonoscopia auxilia para identificar qual a parte do intestino, colón ou ânus se encontra mais afetada. Na histologia será identificado perda da estrutura das criptas e presença de células inflamatórias na lâmina própria. Novas técnicas de imagem como a cápsula endoscópica podem ser utilizadas se ainda houver dúvidas durante a avaliação das imagens; exames de imagem como tomografia computadorizada e ressonância magnética permitem o rastreio de possíveis complicações como perfurações, abcessos e isquemia (Barros et al., 2020).

Diestel et a., (2012) descreve em sua pesquisa orientações dietéticas de acordo com a fase de atividade da doença. Na fase ativa é informado que: 
"Nesta fase é importante que a alimentação auxilie no controle dos sintomas como diarreia, dor abdominal, distensão e previna ou reverta a perda de peso através do uso de suplementos nutricionais adequados. [...] O teor de fibras insolúveis e resíduos (lactose, por exemplo) deve ser restrito e a alimentação deve ser fracionada em seis a oito refeições ao dia, contendo pouco volume" (Diestel et al., 2012).

Na fase de remissão da doença Diestel et al., (2012) descreve em seu estudo que:

"Com a melhora clínica do paciente e o início da fase de remissão, podem ser incluídos os carboidratos simples como a sacarose (em quantidade moderada) e a lactose (progressivamente, caso nao exista intolerancia), deve-se aumentar gradativamente o conteudo de fibras totais e insoluveis da dieta, mantendo-se moderado o teor de gordura (especialmente de ácidos graxos poliinsaturados omega-6). As calorias devem ser adequadas ao estado nutricional do paciente" (Diestel et al., 2012).

Em casos moderados de DC ou RCU, usa-se imunomoduladores e imunossupressores, o mais utilizado é a Azatioprina, caso essa medicação não faça efeito, o metotrexato é utilizado. Ao longo do tratamento, deve ser acompanhado de perto o uso dessas medicações, fazendo exames laboratoriais e verificando em especial os leucócitos, pois essas medicações possuem alguns efeitos colaterais, principalmente se for utilizado à longo prazo e aumenta os riscos de infecções. (Dewulf $e t$ al., 2007).

Por esse motivo, a dietoterapia e imunonutrição em conjunto, servem para prevenir as carências nutricionais ou ajustar a deficiência delas. Em casos de Fase Ativa da doença ou conhecida como crise, a alimentação saudável é indispensável para que o controle seja obtido, é preferível que evite lacticínios, alimentos que possuem muita gordura, comidas que contenham pimenta, evitar de qualquer forma frituras, comidas com molhos e extratos, consumir o mínimo possível de açúcar branco e preferir adoçantes do tipo stevia. Adoçantes do tipo xilitol não são recomendados, pois podem aumentar as idas ao banheiro, assim como as fibras que também não são boas alternativas nesse período. Evitar alimentos ou bebidas que possam produzir gases, como cafeína, refrigerante e bebidas gaseificadas. Nesse período é aconselhável que o glúten seja evitado, pois podem causar inchaços e aumentar as diarreias em alguns pacientes. Após o controlar, é possível voltar a consumir esses alimentos aos poucos. Não é necessário consumi-los, mas se o paciente desejar e tiver fase de remissão, pode ser oferecido (Castro, 2016).

\subsection{Qualidade de vida}

\subsubsection{Questões socioemocionais implicadas}

Ao lidar com sintomas desagradáveis, tais como fadiga, dor, diarreia crônica, desenvolvimento de fístulas entéricas ou perianais, quadros dermatológicos, articulares, oftalmológicos, além da eventual necessidade de cirurgias abdominais e ostomias, as vítimas são submetidas a um aumento de grau de incomodidade e estresse, por isso, ficam sujeitos a expandir crises de depressão e ansiedade. (Falcão \& Martinelli, 2016.)

Algumas das manifestações psicossomáticas, definidas pela Classificação Internacional de Doenças (CID-10) como sintomas psicológicos associados a transtornos não psiquiátricos, podem ser evidenciados em grupos de indivíduos acometidos pelas Doenças Inflamatórias Intestinais em vários estudos (Falcão \& Martinelli, 2016.)

Estudos que trazem relatos de pacientes demonstram que essas doenças inflamatórias do intestino, ao afetarem o estado emocional dos indivíduos, impede-os de realizem suas atividades básicas, por conta das cólicas abdominais, gases e diarreias. Essas intercorrências podem gerar constrangimento, estresse e ansiedade diante dos eventos sociais comprometendo a vida profissional com o afastamento do trabalho, e às vezes chegando até mesmo ao isolamento social, que pode resultar em depressão (Goodwin \& Stein, 2002; Jones \& Maganti, 2004; Levy \& Cols, 2006; Nakao et al., 2007; Silva et al., 2006). 


\subsubsection{Melhora da qualidade de vida}

Um estudo transversal feito em um ambulatório multidisciplinar de referência para tratamento de doenças inflamatórias intestinais do Vale do Itajaí - UNIVALI, onde os pacientes recebem acompanhamento médico, nutricional e psicológico, os pacientes avaliados responderam ao protocolo sociodemográfico e clínico, ao instrumento de avaliação de qualidade de vida IBDQ e ao Short Form36Health Survey (SF-36) e avaliação entre questionários por meio da Correlação de Pearson. Após os dados serem analisados por meio da estatística descritiva, o estudo mostrou que os principais domínios e componentes que trouxeram impacto negativo na qualidade de vida dos entrevistados foram aqueles relacionados aos aspectos emocionais e sintomas sistêmicos da inflamação. Contudo, o acompanhamento multiprofissional desses pacientes, por ser realizado em ambulatório, envolvendo uma equipe de especialistas em gastroenterologia, coloproctologia, nutrição e psicologia, implica resultados positivos na qualidade de vida. (ficagna, 2020)

Outro estudo transversal, realizado no mesmo ambulatório interdisciplinar em Itajá-SC, coletou dados sociodemográficos, tais como: idade, sexo e estado civil, dados socioeconômicos: escolaridade, renda familiar e dados da doença: tipo (DC; RCUI); e atividade da doença (remissão; em atividade). E, realizou perguntas abertas e fechadas, com respostas dicotômicas e de única ou múltipla escolha, sobre o consumo e restrições alimentares, sentimentos relacionados às modificações pós-diagnósticas, bem como reflexos dessas mudanças nas dimensões psicossociais dos participantes. E concluiu que as mudanças alimentares e psicossociais parecem iminentes ao diagnóstico de DII, já que a grande maioria dos pacientes restringe algum alimento e sofreu alguma modificação psicossocial em sua vida após o desenvolvimento da doença. O consumo de álcool, refrigerante, leite e derivados e alimentos gordurosos foram os alimentos/bebidas mais evitados pelos pacientes da pesquisa e a doença pareceu ter gerado impacto socioemocional e profissional na vida dos pacientes (Batista $e t a l$, 2018).

\section{Conclusão}

A contribuição da nutrição no tratamento dos pacientes com Doenças Inflamatórias Intestinais é notória, correlacionando a sua aplicação com a melhora da qualidade de vida desses indivíduos. Descrever quais doenças fazem parte desse grupo e qual o manejo adequado ligado a nutrição é fundamental para o adequado tratamento terapêutico, assim como, o seu impacto na melhora dos pacientes.

Levando em consideração a seleção ao acaso dos artigos, observou-se que, as questões relacionadas a epidemiologia, com relação às Doenças Inflamatórias Intestinais, ainda são incipientes e, portanto, pouco discutida na literatura médica, de um modo geral.

Estudos mostram que a correlação direta da nutrição com a melhoria da qualidade de vida dos pacientes acometidos pelas doenças inflamatórias intestinais ainda possui escassez, porém, através das análises do tratamento com a equipe multidisciplinar é possível observar uma contribuição no quadro de estabilidade dos pacientes. $\mathrm{O}$ atendimento interdisciplinar, o olhar humanizado e integral dos profissionais da saúde para com os pacientes se faz necessário, assim como o desenvolvimento de novos estudos voltados para a temática. Dessa forma, trata-se de uma temática, cuja pesquisa deve ser mais estimulada na academia.

\section{Referências}

Almeida, M. F., Barata R. B., Montero C. V., \& Silva, Z. P. (2002). Prevalência de doenças crônicas auto-referidas e utilização de serviços de saúde. PNAD/1998, Brasil. Ciência e Saúde Coletiva, 7(4), 743-756.

AMBCFM - Associação Médica Brasileira e Conselho Federal de Medicina. (2008). Projeto diretrizes. Doença de Crohn intestinal: manejo. Sociedade Brasileira de Coloproctologia Colégio Brasileiro de Cirurgia Digestiva Sociedade Brasileira de Patologia Colégio Brasileiro de Radiologia. 
Andrade, A. C. M., Santana, G. O., Santos R. R., Guedes, J. C., \& Lyra, L. G. C. (2005). Perfil da doença de Crohn fistulizante em atividade em dois serviços universitários em Salvador - Bahia. Revista Brasileira de Coloproctologia, 25(3), 241-248.

Añez, M., Fuenmayor, M., \& Romero, G. (2012). Enfermedad inflamatoria intestinal: rectocolitis úlcerosa idiopática y enfermedad de Crohn. Revista GEN. $66(3), 197-206$

Barros, G. V. N., Silva, T. S. S., Brito, A. P. S. O., Garcia, H. C. R., \& Maneschy, R. B. (2020). Métodos diagnósticos e terapêuticos das doenças inflamatórias intestinais: revisão sistemática. Para Res Med J. 4, e42. 10.4322/prmj.2019.042.

Batista, T. M., Barretta, C., Matos, C. H., Bobato S. T., Malluta, E. F., Scolaro, B. L., Mello, M. K. M., \& Specht, C. M. (2018). Mudanças no consumo alimentar e nas condições psicossociais geradas pela doença inflamatória intestinal. Revista Brasileira de Tecnologias Sociais. Artigos 10.14210/rbts.v5.n1.p48-58, RBTS - Itajaí - 5(1) https://siaiap32.univali.br/seer/index.php/rbts/article/view/13401/7611 .

Baumgart, D. C, \& Sandborn, W. J. (2012). Crohn's disease. Lancet. 380:1590-1605.

Beyer, P. L. (2010). Tratamento médico nutricional para doenças do trato gastrointestinal inferior. In: Mahn, L. K., \& Escott-Stump, S. Alimentos, Nutrição e Dietoterapia. 12 ed. Rio de Janeiro: Elsevier, 689-695.

Biondo-Simões, M. L. P., Mandelli, K. K., Pereira, M. A. C., \& Faturi, J. L. (2003). Opções terapêuticas para as doenças inflamatórias intestinais: revisão. Rev Bras Coloproct.

British Society Of Gastroenterology. (2003). Guidelines for the management of inflammatory bowel disease. BSG.

Bya LM; Krueger GRF. (2007). Sistema gastrointestinal. In: Bya, L. M., Krueger, G. R. F. Atlas de patologia humana de Netter. Artmed, 2007. 114-115.

Cardozo, W. S., \& Sobrado, C. W. (2015). Doença inflamatória intestinal: Diagnóstico diferencial na doença inflamatória intestinal. (2a ed.), Manole.

Castro, L. S. D. (2016). Alimentação de qualidade para portadores de Doenças Inflamatórias Intestinais (DII). Revista Conexão Eletrônica: 13. Três LagosMS.

Cavalcante, R. M. S., Moura, M. S. B., Braz, D. C., \& Nogueira, N. N. (2020). Retocolite ulcerativas e citocinas: uma revisão da literatura. Research, Society and Development, 9(9), e314997145.http://dx.doi.org/10.33448/rsd-v9i9.7145.

Cruz, V. A., Yamaguchi, L., Ribeiro, C. N., Magalhães, V. O., Rego, J., \& Silva, N. A. (2012). Retocolite ulcerativa e artrite reumatoide: uma rara associação - relato de caso. Rev Bras Reumatol, 52(4), 645-650.

Dewulf, N. L. S., Monteiro, R. A., Passos, A. D. C., Vieira, E. M., Troncon, L. E. A. (2007). Adesão ao tratamento medicamentoso de pacientes com doenças inflamatórias intestinais acompanhados no ambulatório de um hospital universitário. Artigos Originais • Arq. Gastroenterol. 44(4).

Diestel CF; Santos MC; Romi MD. (2012). Tratamento Nutricional nas Doenças Inflamatórias Intestinais. Revista do Hospital Universitário Pedro Ernesto, UERJ.

Diretrizes Práticas da Organização Mundial de Gastroenterologia. (2009). Doença Inflamatória Intestinal: uma perspectiva global.

Ekbom, A., Helmick, C., Zack, M., Adami, H. O. (1991). The epidemiology of inflammatory bowel disease: a large, population-based study in Sweden. Gastroenterology, 100, 350-358.

Falcão, L. T. M., \& Martinelli, V. F. (2016). Associação de doença inflamatória intestinal com ansiedade e depressão: avaliação dos fatores de risco. GED gastroenterol endosc dig. 35(2), 52-58.

Farrukh, A., \& Mayberry, J. F. (2014). Inflammatory bowel disease in hispanic communities: a concerted south american approach could identify the etiology of Crohn's disease and ulcerative colitis. Arq Gastroenterol. 51(3), 271-275.

Ficagna, G. B., et al. (2020). Qualidade de vida dos pacientes de um ambulatório multidisciplinar de doenças inflamatórias intestinais. Arq. Gastroenterol. $57(1), 8-12$.

Frances, D., Monahan, F., \& Sharon, A. (2010). Problemas do Intestino. In: Monahan, F., Sands, J. K., Neighbors, M., \& Marek, J. F.; Green, C.J. Enfermagem médico-cirúrgica: perspectivas de saúde e doença. 8 ed. Loures, Portugal: Lusodidacta, 1284-1291.

Goodwin, R. D., \& Stein, M. B. (2002). Generalized anxiety disorder and peptic ulcer disease among adults in the United States. Psychosom Méd.

Granada, E., Breilh, J. (1998). Saúde na sociedade. Cortez Editora/ Abrasco. Brasil.

Habr-Gama, A, Cerski, C. T. S., Moreira, J. P. T., et al. (2008). Doença de Crohn intestinal: manejo. Diretrizes em Foco. Revista Associação Medicina Brasileira. 57(1), 10-13.

Hanauer, S. B. Inflammatory bowel diseases. ACP Medicine. 2009.

Hardt, M. R., et al. (2012). Epidemiological profile of 175 patients with Crohn's disease submitted to biological therapy. J Coloproctol. (Rio J). 32(4), 395401.

Jones, M. P. \& Maganti, K. (2004). Symptoms, gastric function, and psychosocial factors in functional dyspepsia. J Clin Gastroenterol.

Levy, R., K., Olden, K., Naliboff, B., Bradley, L., Francisconi, C., Drossman, D., \& Creed, F. (2006). Psychosocial Aspects of the Functional Gastrointestinal Disorders. Gastroenterology. 
Research, Society and Development, v. 10, n. 15, e236101523029, 2021

(CC BY 4.0) | ISSN 2525-3409 | DOI: http://dx.doi.org/10.33448/rsd-v10i15.23029

Lima, F. D. V. D., et al. (2012). Oscilações do humor em pacientes com doença de Crohn: Incidencia e fatores associados. 481-488.

Maranhão, D. D. A., Viera, A., Campos, T. (2015). Características e diagnóstico diferencial das doenças inflamatórias intestinais. JBM, Vol. $103(9)$, 01.

Nakao, H., Konishi, H., Mitsufuji, S., Yamauchi, J., Yasu, T., Taniguchi, J., Wakabayashi, N., Kataoka, K., \& Okanoue, T. (2007). Comparison of clinical features and patient background in functional dyspepsia and peptic ulcer. Dig Dis Sci.

Oliveira, F. M., Emerick, A. P. C., Soares, E. G. Aspectos epidemiológicos das doenças intestinais inflamatórias na macrorregião de saúde leste do Estado de Minas Gerais. Universidade Federal de Campina Grande, Unidade acadêmica de ciências da vida. Paraíba, 15(1):1031-1037.

Oliveira, F. M.; Emerick, A. P. C.; Soares, E. G. Aspectos epidemiológicos das doenças intestinais inflamatórias na macrorregião de saúde leste do Estado de Minas Gerais. Ciência \& Saúde Coletiva, 15(1), 1031- 1037, 2010.

Ordás, J., Eckmann, L., Talamini, M., Baumgart, D. C., et al. Ulcerative Colitis. The Lancet. 380(9853):1606- 1619.

Papacosta, N. G., Nunes, G. M., Pacheco, R. J., Cardoso, M. V., Guedes, V. R. Doença de Crohn: um artigo de revisão. Revista de patologia do Tocantins, 2017.

Pinho, Mauro. A biologia molecular das Doenças Inflamatórias Intestinais. Ver.bras Coloproct, 28, 119-123, 2008.

SBC - Sociedade Brasileira de C oloproctologia; Colégio Brasileiro de Cirurgia Digestiva; Sociedade Brasileira de Patologia; Colégio Brasileiro de Radiologia. DIRETRIZES EM FOCO. Doença de Crohn intestinal: manejo. Rev Assoc Med Bras 57(1):10-13.

Santos, A. L. C., Dias, B. C. O., Silva, K. A., \& Ferreira, J. C. S. (2021). Terapia nutricionais nas doenças inflamatórias intestinais: Doença de Crohn e Retocolite Ulcerativa. Research, Society and Development, 10(7), e 11410716660, http://dx.doi.org/10.33448/rsd-v10i7.16600.

Silva, A. F. D., Schieferdecker, M. E. M., \& Amarante, H. M. B. D. S. (2011). ABCD Arq. Bras. Cir. Dig: Ingestão alimentar em pacientes com Doenças Inflamatórias Intestinais, 204-209.

Silva, R. A., Pinheiro, R. T., Silva, R. A., Horta, B. L., Moraes, I., \& Faria, A. D. (2006). Dispepsia funcional e depressão como fator associado. Artigos Originais $\cdot$ Arq. Gastroenterol. 43(4).

Souza, M. M., Belasco, A. G. S., Aguilar-Nascimento, J. E. (2208). Perfil epidemiológico dos pacientes com doença inflamatória intestinal no Estado de Mato Grosso. Rev Bras Coloproct. 28, 324-8.

Stevens, A, \& Lowe, J. S. (2202). Patologia. (2a ed.), Manole.

Yashiro, M. (2014). Ulcerative colitis-associated colorectal câncer. World J Gastroenterol. 20(44), 16389 - 16397.

Zanardi, M. M. M., \& Nowacki, L. (2018). Doenças Inflamatórias Intestinais - Uma Revisão Bibliográfica. Revista Eletrônica Biociências, Biotecnologia e Saúde, 20. 